\title{
Competencia digital de los profesores de inglés en enseñanza primaria del sureste de México
}

\author{
Digital Competence of Elementary School English Teachers in Southeast
}

Mexico

\section{Competência digital de professores de inglês na educação primária no} sudeste do México

Sergio Humberto Quiñonez Pech

Universidad Autónoma de Yucatán, México

sergio.quinonez@correo.uady.mx

https://orcid.org/0000-0001-5220-9912

\section{Resumen}

Debido a los diversos avances tecnológicos, las tecnologías de información y comunicación (TIC) han transformado la forma en cómo se aprende un idioma. En la actualidad, la educación implementa modelos pedagógicos innovadores que permiten incorporar la tecnología a los diversos procesos de enseñanza y aprendizaje del idioma inglés. Por lo antes mencionado, este artículo describe los resultados de una encuesta sobre el nivel de competencia digital que tienen los profesores de inglés en enseñanza primaria a partir de las dimensiones propuestas por los autores Usher y Pajares (2007), y también se identifican las variables que modifican dicha competencia. El estudio fue cuantitativo de tipo descriptivo y transversal. Los participantes fueron profesores que estuvieron en el Programa de Inglés en Educación Básica de Yucatán (PIEBY). Los resultados demostraron que $81 \%$ de los profesores tienen un nivel de competencia digital bajo. Otro hallazgo importante que se obtuvo fue que se identificaron las dimensiones técnica, pedagógica y de comunicación como necesidades para ser atendidas a través de cursos de capacitación con un nivel básico. Asimismo, se evidenció que las variables de edad y grado máximo de estudios influyen en el nivel de la competencia digital de los profesores. Entre las conclusiones a destacar está que las TIC son herramientas muy importantes para la enseñanza del idioma inglés, ya que ayudan a despertar el interés de los 


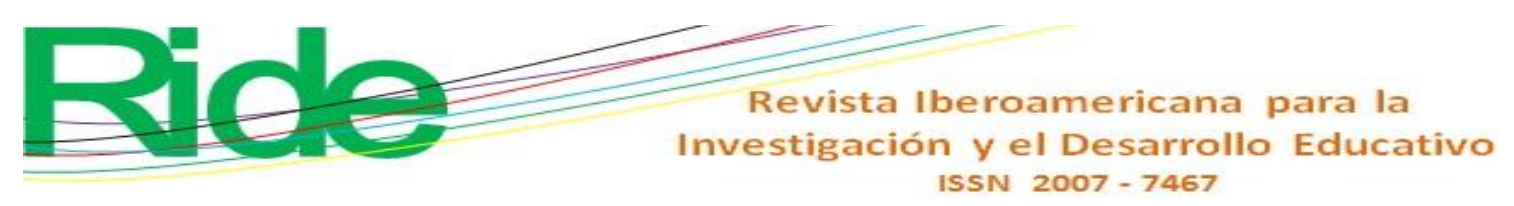

estudiantes, permite crear un ambiente interactivo, brinda muchos recursos de apoyo y contribuyen a que las clases sean atractivas e innovadoras.

Palabras clave: competencia digital, enseñanza del idioma inglés, enseñanza primaria, TIC.

\section{Abstract}

Given the various technological advances, information and communication technologies (ICT) have transformed the way a language is learned. Nowadays, education is implemented through innovative pedagogical models which allow technology to incorporate the various processes of teaching and learning of the English language. For the aforementioned reasons, this article describes the results of a survey on the level of digital competence that English teachers have in elementary school, based on the dimensions proposed by the authors Usher and Pajares (2007), as well as the variables that influence it. The study was quantitative, descriptive and transversal. The participants were teachers who were in the "Program of English in Basic Education in Yucatán" (PIEBY, by its acronym in Spanish). The results demonstrated that $81 \%$ of the teachers have a low level of digital competence. Another important finding that was acquired from the study was that the technical, pedagogical and communication dimensions were identified as necessities to be addressed through training courses on a basic level; likewise, it was proven that the age and maximum degree of study variables influence the level of digital competence in teachers. Among the conclusions to emphasize is that ICT are very important tools for the teaching of the English language, as they help awaken the interest in students, they allow to create an interactive environment, they provide many support resources, and they favor the classes to be attractive and innovative.

Keywords: digital competence, english language teaching, elementary education, ICT.

\section{Resumo}

Devido a vários avanços tecnológicos, as tecnologias de informação e comunicação (TIC) transformaram a forma como uma língua é aprendida. Atualmente, a educação implementa modelos pedagógicos inovadores que permitem incorporar a tecnologia nos diversos processos de ensino e aprendizagem da língua inglesa. Diante do exposto, este artigo descreve os resultados de uma pesquisa sobre o nível de competência digital que professores de inglês possuem no ensino fundamental com base nas dimensões propostas pelos autores Usher e Pajares (2007), e as variáveis também são identificadas. que modificam a referida competição.

$\mathrm{O}$ estudo foi quantitativo, descritivo e transversal. Os participantes foram professores do Programa de Inglês na Educação Básica de Yucatán (Pieby). Os resultados mostraram que 81\% 


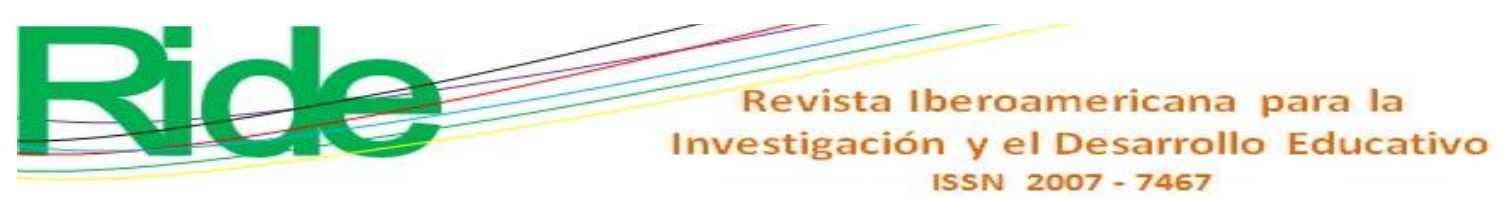

dos professores possuem baixo nível de competência digital. Outra constatação importante obtida foi que as dimensões técnica, pedagógica e de comunicação foram identificadas como necessidades a serem atendidas por meio de cursos de formação com nível básico. Da mesma forma, evidenciou-se que as variáveis idade e grau máximo de estudos influenciam no nível de competência digital dos professores. Dentre as conclusões a serem destacadas, está que as TICs são ferramentas muito importantes para o ensino da Língua Inglesa, pois ajudam a despertar o interesse dos alunos, permitem a criação de um ambiente interativo, fornecem diversos recursos de apoio e contribuem para tornar as aulas atrativas. e inovador.

Palavras-chave: competência digital, ensino de língua inglesa, educação primária, TIC.

Fecha Recepción: Abril 2020

Fecha Aceptación: Septiembre 2020

\section{Introducción}

En la actualidad, las tecnologías de información y comunicación (TIC) representan una herramienta imprescindible en el ámbito de la educación ya que apoyan y transforman los procesos de enseñanza-aprendizaje, lo que sin duda favorece la innovación en los diversos niveles educativos (Cabrera, Sánchez y Rojas, 2016). Valdés, Angulo, Urías, García y Mortis (2011) afirman que el uso de las tecnologías en los programas educativos es relevante porque promueven la calidad educativa y facilitan el aprendizaje de los estudiantes.

Con la intención de apoyar y desarrollar la competencia digital, así como desarrollar las habilidades del lenguaje y comunicación tanto en estudiantes como en profesores de enseñanza primaria, el Gobierno federal, a través de la Secretaría de Educación Pública [SEP] (11 de octubre de 2017), ha desarrollado una serie de programas destinados a impulsar la inserción y utilización de las TIC en el sistema educativo. En esa misma línea, el Plan Estatal de Desarrollo de Yucatán 2018-2024 (Gobierno del Estado de Yucatán, 30 de marzo de 2019) establece que se debe "promover los avances y beneficios de la ciencia y tecnología en los municipios y en todos los niveles educativos" (p. 329).

La enseñanza y aprendizaje del idioma por medio de la tecnología ha traído grandes beneficios. Por medio de ella se tiene acceso a materiales auténticos como periódicos en línea, webcasts, podcasts e incluso videos en la lengua estudiada (Swapna y Tammelin, 2008). Asimismo, permite que los profesores desarrollen su competencia digital, la cual consiste en el uso seguro y crítico de las tecnologías de la información y el dominio de las TIC para el trabajo, el ocio y la comunicación (Pérez y Monteza, 2013).

Una investigación acerca de las percepciones de los profesores y estudiantes sobre el uso de la tecnología para la enseñanza y aprendizaje del idioma inglés reportó que la tecnología genera en los estudiantes un rol activo e interactivo y a los profesores les permite diversificar 


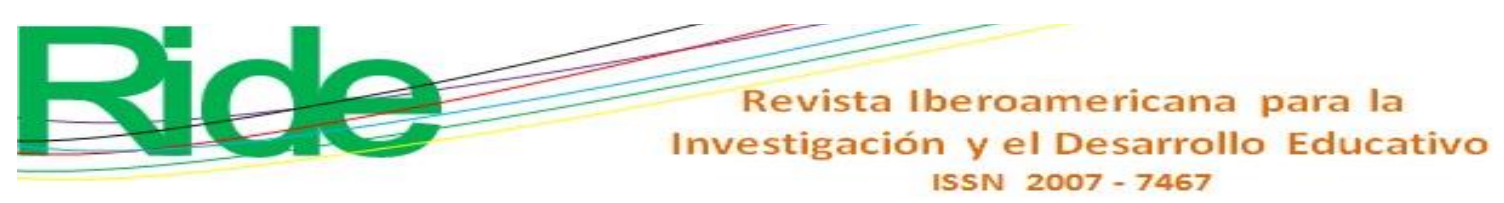

sus estrategias durante el proceso de enseñanza y aprendizaje (Díaz y Jansson, 2011). Por otra parte, García y Rey (2013) realizaron una investigación cualitativa para conocer los puntos de vista de los profesores acerca del uso de la tecnología en la enseñanza de inglés. Entre los resultados se encontró que los profesores de inglés sí están conscientes de la importancia del uso de las TIC y diseñan actividades que involucran la tecnología para desarrollar principalmente habilidades receptivas; sin embargo, dejan a un lado las productivas, es decir, la escritura y el habla.

A pesar de todo lo positivo que puede significar el uso de las TIC en el ámbito educativo, Torres y Valencia (2013) señalan que los profesores de educación básica, independientemente de la disciplina, poseen insuficiente conocimiento tecnológico y no logran integrar las TIC como apoyo a la enseñanza, por lo que se centran solo en los aspectos técnicos. En el caso de la enseñanza del idioma inglés, los audios y videos son los más utilizados por los profesores para el desarrollo de la habilidad de escucha (Morchio, 2014); otros recursos tecnológicos como el uso de las plataformas educativas, aplicaciones móviles y las herramientas de la web 2.0, que ayudan a diversificar y ejercitar las cuatro habilidades del idioma inglés, son dejados de lado.

Ante lo mencionado anteriormente, el presente trabajo tiene dos objetivos. Por un lado, describir el nivel de competencia digital de los profesores de inglés en enseñanza primaria a partir de las dimensiones propuestas por los autores Usher y Pajares (2007); por el otro, identificar las variables que influyen en dicha competencia. Debido a que la tecnología es benéfica para la enseñanza del idioma inglés, como ya ha quedado delineado líneas arriba, es importante contar con información del estado actual del nivel de competencia digital de los profesores para que las instituciones correspondientes puedan tomar decisiones en beneficio de las necesidades detectadas.

\section{Marco de referencia}

\section{Las TIC y la enseñanza del idioma inglés}

Hoy en día todos nos encontramos inmersos en la cultura tecnológica. Es utilizada en cada nivel educativo: desde el preescolar hasta el nivel superior. Los estudiantes han desarrollado una fascinación por la tecnología, principalmente por los videos y las redes sociales (Echeburúa, Labrador y Becoña, 2009). Prensky (2010) llama a estas personas nativos digitales. Además de estar acostumbrados a recibir información de una manera verdaderamente rápida, estos nuevos estudiantes son personas multitasking, les gusta realizar varias actividades al mismo tiempo (buscar información con el celular, teclear la información en la computadora, escuchar música en la tableta, etc.), prefieren los gráficos en lugar de los textos, las recompensas permanentes y las estrategias basadas en los videojuegos. 


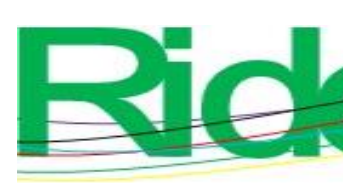

Revista Iberoamericana para la

Investigación y el Desarrollo Educativo

ISSN 2007 - 7467

hojas de trabajo (Chacón y Pérez, 2011; Dudeney y Hockly, 2007). Asimismo, para fomentar un aprendizaje polifacético e innovador del idioma inglés y para que los estudiantes se expresen de manera más articulada y creativa en un ambiente tecnológico e interactivo, utilizan las redes sociales y videollamadas (Buzzetto, 2012; González, 2012; González y Mayora, 2013).

\section{Competencia docente para la enseñanza del idioma inglés}

Las reformas educativas nacionales han estado promoviendo desde hace tiempo el aprendizaje del idioma inglés, así como un cambio de las prácticas docentes para favorecer la movilización de conocimientos, habilidades y actitudes comunicativas de la lengua extranjera (SEP, 2011). En la actualidad, un docente de enseñanza del idioma inglés no solo debe ser competente desde el punto de vista linguí́stico, sino también desde el pedagógico y metodológico, ya que debe poder dirigir el proceso de enseñanza-aprendizaje para el desarrollo de las cuatro habilidades del idioma inglés (leer, escribir, escuchar y hablar). Más aún: también debe ser capaz de implementar técnicas y procedimientos de enseñanza en el aula de acuerdo con los estilos y estrategias de aprendizaje de sus estudiantes (Páez, 2001).

Con base en la propuesta de Perrenoud (2004), la SEP (2010) estableció las competencias que debe poseer un profesor de enseñanza del idioma inglés:

1) Domina los contenidos de enseñanza del currículo del idioma inglés (saber).

2) Domina los referentes, funciones y estructura del idioma inglés (saber).

3) Implementa actividades de aprendizaje que favorecen la movilización de conocimientos, habilidades y actitudes que intervienen en el desarrollo del idioma inglés (saber hacer).

4) Crea, adapta y explota de manera creativa materiales empleando diversos fundamentos teóricos, métodos y estrategias de enseñanza de segundas lenguas para favorecer el aprendizaje del idioma inglés (saber hacer).

5) Promueve los valores de la ciudadanía y de los derechos humanos de las culturas de la segunda lengua (saber convivir).

6) Atiende de manera adecuada la diversidad cultural, lingüística, estilos de aprendizaje y puntos de partida de los estudiantes (saber ser).

7) Trabaja de forma colaborativa y crea redes académicas en la docencia en beneficio de la enseñanza del idioma inglés (saber convivir).

8) Organiza su propia formación continua y se involucra en procesos de desarrollo personal y autoformación profesional (saber ser).

9) Incorpora las tecnologías de la información y la comunicación en los procesos de enseñanza-aprendizaje del idioma inglés (saber hacer). 


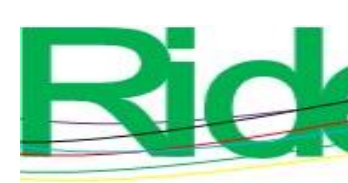

Revista Iberoamericana para la Investigación y el Desarrollo Educativo ISSN $2007-7467$

Como se puede observar en la propuesta anterior, y teniendo en cuenta el objetivo de este trabajo, la enseñanza del idioma inglés y el uso de las TIC convergen de una manera precisa y eficaz dentro y fuera del aula (Rodríguez y Gómez, 2017). De esta forma, en el proceso de enseñanza y aprendizaje presencial o virtual del inglés, se conjugan el uso de las TIC y las nuevas metodologías pedagógicas; esto hace que se necesite en la actualidad de profesores con una competencia digital sólida que impacte al ámbito educativo, con el fin de ofrecer calidad e innovación en la formación de una segunda lengua como es el idioma inglés.

\section{Competencia digital y la enseñanza del idioma inglés}

Uno de los desafíos de cualquier profesor hoy en día es el de centrar la enseñanza en el estudiante y volverlo más activo y reflexivo durante el proceso de su aprendizaje; las tecnologías han servido de apoyo en dicho proceso. Es por eso por lo que el profesor de inglés ha tenido que cambiar su forma de enseñar; ahora es alguien que necesita ser competente en el diseño y uso eficiente de las TIC para la didáctica del idioma meta. Asimismo, debe poseer habilidades tecnológicas para diseñar ambientes de aprendizaje apropiados para el estudiante del nuevo siglo (Norton y Wiburg, 1998 citado en Villalba 2008). Estos mismos autores afirman que el profesor de inglés tiene que ser capaz de crear espacios para la interacción y uso del lenguaje meta, así como poder adecuar las herramientas TIC para transmitir de una manera efectiva el conocimiento. Butler (2011), por su parte, indica que, en este nuevo rol donde el profesor es un guía para el aprendizaje, si este utiliza la tecnología como apoyo a la enseñanza del idioma inglés además puede desarrollar su competencia digital y fomentar en los estudiantes habilidades lingüísticas y tecnológicas.

Gutiérrez (2014) define la competencia digital de la siguiente forma:

Valores, creencias, conocimientos, capacidades y actitudes para utilizar adecuadamente las tecnologías, incluyendo tanto los ordenadores como los diferentes programas e Internet, que permiten y posibilitan la búsqueda, el acceso, la organización y la utilización de la información con el fin de construir conocimiento" (p. 54).

Fainholc, Nervi, Romero y Halal (2015) recomiendan el desarrollo de la competencia digital a través de la generación de entornos personales de aprendizaje virtuales y el uso de los dispositivos móviles; lo interesante de hacer esto es que no solo se abarca el ámbito tecnológico, sino que también se desarrollan las capacidades de comunicación, aprendizaje y gestión de la información, que son importantes para la enseñanza del idioma inglés. En suma, en los nuevos escenarios de enseñanza-aprendizaje, el profesor de inglés debe saber comunicarse, relacionarse y colaborar en ambientes digitales (el uso de redes sociales, plataformas, blogs, 


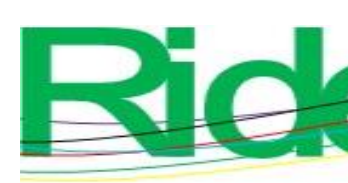

Revista Iberoamericana para la

Investigación y el Desarrollo Educativo

ISSN $2007-7467$

etcétera); debe también poder evaluar información de forma crítica para poder generar conocimiento y evidenciarlo en funciones superiores de pensamiento.

Cabe señalar que un profesor con un nivel alto de competencia digital es capaz de identificar las distintas maneras de representar, recolectar, distribuir, comunicar e interactuar con información adquirida en la Red (Bauman, 2004). Cuando utilizan correctamente las TIC en el aula, demuestran ser capaces de navegar en la Red críticamente. Así, seleccionan información confiable y útil, se apropian del lenguaje tecnológico, incluyen actividades significativas usando las TIC (como juegos, simulaciones, resolución de problemas, estudios de caso), son capaces de desarrollar multitareas y una secuencia de clase incluyendo recursos tecnológicos que tengan un fin, objetivo o criterio pedagógico (Fainholc et al., 2015).

Es por lo antes mencionado que algunas instituciones han establecido estándares de calidad para profesores y el uso de la tecnología en el aula. En 2008, la International Society for Technology in Education (ISTE, por sus siglas en inglés) estableció los siguientes estándares para los profesores: creatividad e innovación, búsqueda y manejo de la información, comunicación y colaboración, pensamiento crítico, solución de problemas y toma de decisiones, ciudadanía digital, manejo de la tecnología y sus conceptos (ISTE, 2008). Por su parte, Usher y Pajares (2007) construyeron una escala para la percepción de la competencia digital, de la cual cuatro de sus dimensiones se utilizaron para este estudio (ver tabla 1).

Tabla 1. Descriptores de las dimensiones de la competencia digital

\begin{tabular}{|l|l|}
\hline $\begin{array}{l}\text { Dimensiones } \\
\text { competencia digital }\end{array}$ & Descriptor \\
\hline Técnico & $\begin{array}{l}\text { El profesor aplica los principales medios tecnológicos de } \\
\text { su entorno en el proceso de enseñanza. }\end{array}$ \\
\hline Pedagógico & $\begin{array}{l}\text { El profesor planifica la creación de experiencias de } \\
\text { aprendizaje auténticas con base al uso de las TIC. }\end{array}$ \\
\hline Comunicación & $\begin{array}{l}\text { El profesor crea experiencias para hacer contribuciones } \\
\text { en las redes sociales y se trabaje de manera colaborativa. }\end{array}$ \\
\hline Actitud & $\begin{array}{l}\text { El profesor cuenta con la habilidad de organizar y } \\
\text { gestionar las TIC haciendo un uso responsable de estos } \\
\text { elementos. }\end{array}$ \\
\hline
\end{tabular}

Fuente: Usher y Pajares (2007) 


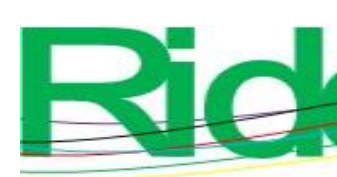

Revista Iberoamericana para la Investigación y el Desarrollo Educativo

ISSN 2007 - 7467

Es importante recordar que el profesor debe de utilizar las TIC dentro y fuera del aula en beneficio del proceso enseñanza-aprendizaje; es por esto por lo que Lorber (1977) hace énfasis en la necesidad de desarrollar la competencia digital, ya que esto garantiza el éxito del ámbito educativo.

\section{Método}

La presente investigación tuvo un enfoque cuantitativo con alcance descriptivo, ya que se procuró determinar cómo se manifiesta cierto fenómeno en un contexto determinado (Hernández, Fernández y Baptista, 2013). De igual forma, es considerada transeccional, debido a que la medición y recolección de los datos se realizó en un solo momento en el tiempo (Cozby y Bates, 2015). Por último, el diseño del estudio fue de tipo encuesta; permitió describir la opinión y las actitudes de las personas mediante el registro y análisis de los datos que proporcionaron durante el proceso de la investigación (Isaac y Michael, 1995). Las fases que integraron este estudio fueron: planteamiento del objetivo, planificación de la muestra, recolección de los datos y análisis e interpretación de los datos (Arnau, 1995).

Siguiendo las fases del estudio tipo encuesta, en su primera fase se plantearon los objetivos que guiarían el estudio: describir el nivel de competencia digital de los profesores de inglés en enseñanza primaria a partir de las dimensiones propuestas por los autores Usher y Pajares (2007) e identificar las variables que juegan un papel primordial en esta.

Para la segunda fase (para la selección de los participantes), se utilizó una muestra no probabilística de tipo intencional (Casal y Mateu, 2003), pues solamente se tomaron en cuenta a los profesores de inglés de enseñanza primaria que habían participado en el Programa de Inglés en Educación Básica de Yucatán (PIEBY). La muestra final estuvo comprendida por 167 profesores de inglés de enseñanza primaria, de los cuales $76 \%(n=127)$ fueron mujeres y $24 \%$ $(n=40)$ fueron hombres. Con respecto a las edades de los participantes, los de mayor frecuencia fueron los que se encontraban entre los 26 a 35 años y los de menor frecuencia los que tenían más de 55 años. En cuanto al grado máximo de estudios, solo $1 \%(n=2)$ de los profesores indicó no contar con el grado de licenciado; $84 \%(n=141)$ mencionó que cuenta con licenciatura, $1 \%(n=2)$ señaló haber cursado una especialización, $13 \%(n=23)$ señaló que cuenta con maestría y solo un profesor aseguró haber concluido un doctorado.

En la tercera fase (recolección de datos), se diseñó el cuestionario denominado Percepción del profesor de enseñanza del idioma inglés respecto a su competencia digital, el cual sirvió para responder a los objetivos planteados en el estudio. Para la base conceptual del instrumento se tomó como referencia el trabajo realizado por Usher y Pajares (2007) y Cabero, Llorente y Marín (2010). 


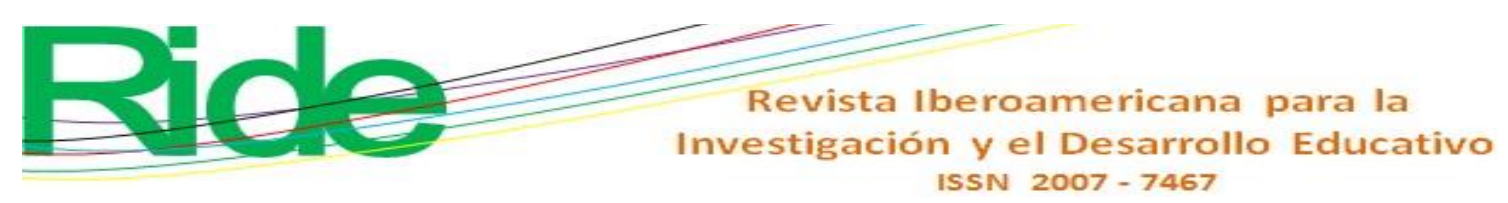

El cuestionario estuvo conformado por dos secciones. La primera tuvo el objetivo de solicitar información general: sexo, edad, experiencia docente, último grado de estudios, etc. La segunda sección, a través de 26 reactivos divididos en cuatro dimensiones: técnica, pedagógica, comunicación y actitud, tuvo como objetivo conocer la percepción del profesor de inglés de enseñanza primaria respecto a su competencia digital.

La escala que se utilizó en el instrumento fue de tipo Likert. Contó con un rango de opciones de respuestas que iba del 1 al 5 , donde $1=$ Nada competente, $2=$ Poco competente, 3 = Medianamente competente, $4=$ Competente y $5=$ Muy competente. Este tipo de escalas constituye uno de los formatos más utilizados cuando se desea preguntar varias cuestiones que comparten las mismas opciones de respuesta (Cea D'Ancona, 2001).

El cuestionario se desarrolló para ser administrado en línea mediante Google Forms. Esta es una herramienta colaborativa de fácil distribución y acceso que garantiza a los usuarios almacenar datos con un alto nivel de seguridad. Gracias a esta herramienta, el instrumento pudo ser contestado desde cualquier dispositivo y lugar; de manera automática, recolectó los datos que se utilizarían en el análisis de los resultados.

Como parte del análisis psicométrico efectuado al instrumento para determinar su confiabilidad y validez, se realizó la prueba de discriminación de reactivos, comparando las puntuaciones en cada uno de estos. Con base en los datos obtenidos, se determinó que a los participantes cuyas puntuaciones estuvieran por arriba del cuartil 75 se les consideraría competentes digitales; en cambio, si obtenían puntuaciones ubicadas por debajo del cuartil 25, se les consideraría limitados en cuanto a su competencia digital. Este proceso estadístico se realizó a través de una prueba $t$ para muestras independientes (tabla 2). 
Tabla 2. Prueba de discriminación de reactivos

\begin{tabular}{|c|c|c|}
\hline Reactivos & $t$ & $\boldsymbol{P}$ \\
\hline $\begin{array}{l}\text { Realiza un documento escrito con un procesador de texto, por ejemplo: } \\
\text { Google Docs, Microsoft Word. }\end{array}$ & -4.00 & 0.000 \\
\hline Maneja alguna plataforma, por ejemplo: Moodle, Edmodo, etc. & -5.94 & 0.000 \\
\hline $\begin{array}{l}\text { Crea o edita imágenes mediante algún programa de diseño gráfico, por } \\
\text { ejemplo: Gimp, Polarr, Hexels, etc. }\end{array}$ & -5.99 & 0.000 \\
\hline $\begin{array}{l}\text { Crea presentaciones multimedia e interactivas mediante algún programa, } \\
\text { por ejemplo: PowerPoint, Prezi, Emaze, PowToon, etc. }\end{array}$ & -7.69 & 0.000 \\
\hline $\begin{array}{l}\text { Busca información en Internet con diferentes navegadores, por ejemplo: } \\
\text { Explorer, Chrome, Mozilla, Opera. }\end{array}$ & -3.58 & 0.001 \\
\hline Desarrolla blogs para sus asignaturas, por ejemplo: Wix. & -7.34 & 0.000 \\
\hline $\begin{array}{l}\text { Utiliza las redes sociales como apoyo a sus asignaturas, por ejemplo: } \\
\text { Facebook, Instagram, Pinterest. }\end{array}$ & -5.93 & 0.000 \\
\hline $\begin{array}{l}\text { Crea videos para sus asignaturas utilizando algún editor de video, por } \\
\text { ejemplo: YouTube Video Editor. }\end{array}$ & -10.23 & 0.000 \\
\hline $\begin{array}{l}\text { Crea podcasts para sus asignaturas mediante editores de audio, por } \\
\text { ejemplo: Audacity. }\end{array}$ & -7.37 & 0.000 \\
\hline $\begin{array}{l}\text { Puede conectar equipos de cómputo y audiovisuales (videoproyector, } \\
\text { reproductor de películas y audio, bocinas, etc.). }\end{array}$ & -6.68 & 0.000 \\
\hline $\begin{array}{l}\text { Sintetiza la información mediante tablas, gráficos o esquemas para } \\
\text { presentar información a sus estudiantes. }\end{array}$ & -5.98 & 0.000 \\
\hline $\begin{array}{l}\text { Implementa diferentes estrategias de enseñanza basadas en TIC que } \\
\text { favorecen el aprendizaje del idioma inglés, por ejemplo: implementación } \\
\text { de juegos interactivos para el aprendizaje de vocabulario en inglés. }\end{array}$ & -11.14 & 0.000 \\
\hline $\begin{array}{l}\text { Utiliza la videoconferencia como apoyo para el aprendizaje del idioma } \\
\text { inglés, por ejemplo: platicar con estudiantes nativos en el idioma. }\end{array}$ & -9.51 & 0.000 \\
\hline $\begin{array}{l}\text { Identifica necesidades en la enseñanza del idioma inglés que pueden ser } \\
\text { abordadas con el uso de las TIC. }\end{array}$ & -10.42 & 0.000 \\
\hline $\begin{array}{l}\text { Promueve diferentes estilos de aprendizaje para el idioma inglés utilizando } \\
\text { las TIC, por ejemplo: visual, auditivo y kinestésico. }\end{array}$ & -8.67 & 0.000 \\
\hline $\begin{array}{l}\text { Diseña diferentes tipos de evaluación (diagnóstica, formativa y sumativa) } \\
\text { para valorar el aprendizaje en el idioma inglés por medio de test en línea, } \\
\text { por ejemplo: Hot Potatoes, ThatQuiz, Google Forms. }\end{array}$ & -8.22 & 0.000 \\
\hline
\end{tabular}




\begin{tabular}{|l|l|l|}
\hline $\begin{array}{l}\text { Explica las ventajas y limitaciones que presentan las TIC para la enseñanza } \\
\text { del idioma inglés. }\end{array}$ & -10.54 & 0.000 \\
\hline $\begin{array}{l}\text { Se comunica con estudiantes por medio de alguna plataforma educativa } \\
\text { fuera del horario escolar, por ejemplo: foro, chat, wiki, etc. }\end{array}$ & -7.96 & 0.000 \\
\hline $\begin{array}{l}\text { Utiliza programas de la web 2.0 (por ejemplo: Google Drive, YouTube, } \\
\text { wikis, blogs, etc.) para compartir información académica en la red con sus } \\
\text { compañeros profesores y estudiantes. }\end{array}$ & -12.59 & 0.000 \\
\hline $\begin{array}{l}\text { Utiliza el correo electrónico para propiciar la práctica del idioma inglés } \\
\text { (por ejemplo: pen pals, escribir textos para practicar lectura y escritura) o } \\
\text { para enviar tareas. }\end{array}$ & -10.84 & 0.000 \\
\hline $\begin{array}{l}\text { Crea espacios de práctica del idioma inglés fuera del salón de clase a través } \\
\text { del uso de las TIC. Por ejemplo: actividades o juegos en páginas web } \\
\text { educativas, proponer algún clip de video o película en inglés, diseñar } \\
\text { actividades online, etc. }\end{array}$ & -15.06 & 0.000 \\
\hline $\begin{array}{l}\text { Desarrolla una actitud abierta y crítica ante las nuevas tecnologías } \\
\text { (contenidos, entretenimiento, etc.). }\end{array}$ & -10.45 & 0.000 \\
\hline $\begin{array}{l}\text { Disposición al aprendizaje continuo y a la actualización permanente. } \\
\text { obtenida de Internet, por ejemplo: propiedad intelectual, licencias de } \\
\text { distribución. }\end{array}$ & -7.37 & 0.000 \\
\hline $\begin{array}{l}\text { Fomenta en sus alumnos el uso de las TIC al pedirles que realicen tareas } \\
\text { que suponga usarlas (un video, una grabación, un blog, etc.). }\end{array}$ & -11.85 & 0.000 \\
\hline Demuestra aspectos éticos y legales al momento de utilizar la información & -7.39 & 0.000 \\
\hline
\end{tabular}

\section{Fuente: Elaboración propia}

Del análisis de los datos observados en la tabla 2, se determinó que los reactivos sí discriminan, ya que el valor de significancia ( $p$ ) es menor a 0.05. Asimismo, se corrió la prueba estadística denominada alfa de Cronbach y se obtuvo un valor de $\alpha=0.908$. Este resultado demostró la confiabilidad del cuestionario. Al respecto, George y Mallery (2003) señalan que un coeficiente de alfa igual o mayor a 0.8 indica que el instrumento es confiable.

La recolección de los datos se realizó en línea, se envió el cuestionario denominado Percepción del profesor de enseñanza del idioma inglés respecto a su competencia digital a través de las cuentas de correo electrónico de los diversos profesores que participaron en el estudio. En el cuerpo del correo se explicaba cuál era el propósito de la investigación; dentro se les solicitaba que contestaran el cuestionario de una forma objetiva y honesta para poder 


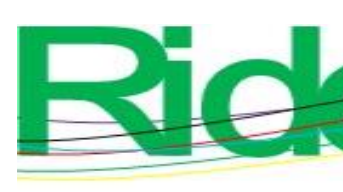

Revista Iberoamericana para la Investigación y el Desarrollo Educativo ISSN $2007-7467$

garantizar la veracidad de los resultados. Por último, se les comunicó que la información obtenida sería confidencial, anónima y su utilización sería únicamente para los fines de investigación establecidos.

En la cuarta fase se realizaron pruebas estadísticas como la obtención de la media, los porcentajes y frecuencias de los datos. Esto con el objetivo de categorizar el nivel de la competencia digital; también se realizó la prueba $t$ de Student para muestras independientes, para determinar si existía o no diferencias entre la competencia digital y la variable sexo. Finalmente, se realizó un análisis de varianza para determinar si existía diferencia entre la competencia digital y las variables grado máximo de estudios, edad y años de experiencia docente.

\section{Resultados}

En esta primera parte de los resultados se indica que el instrumento cuantitativo fue contestado por 167 profesores de inglés de enseñanza primaria: $76 \%$ mujeres y $24 \%$ hombres. Al analizar los resultados obtenidos, se pudo evidenciar que $99 \%$ de los encuestados tenía un teléfono celular inteligente y solo $37 \%$ una computadora de escritorio. Como se puede observar, los profesores cuentan sobre todo con tecnología móvil (ver figura 1).

Figura 1. Porcentaje de los equipos tecnológicos con los que cuenta el profesor de inglés

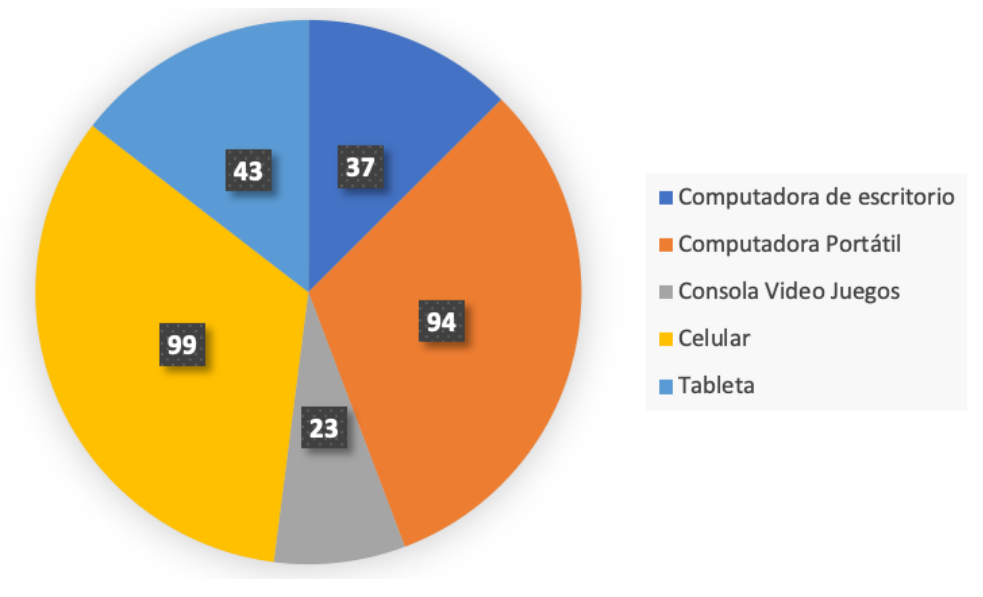

Fuente: Elaboración propia

Otro dato importante es que $96 \%$ de los profesores de inglés usa aplicaciones para reproducir audios y videos en sus dispositivos móviles, lo cual resulta lógico ya que existe una gran variedad de aplicaciones para enseñar inglés que se basan en la reproducción de audios y videos (ver figura 2). 
Figura 2. Porcentaje de las aplicaciones que utiliza el profesor de inglés

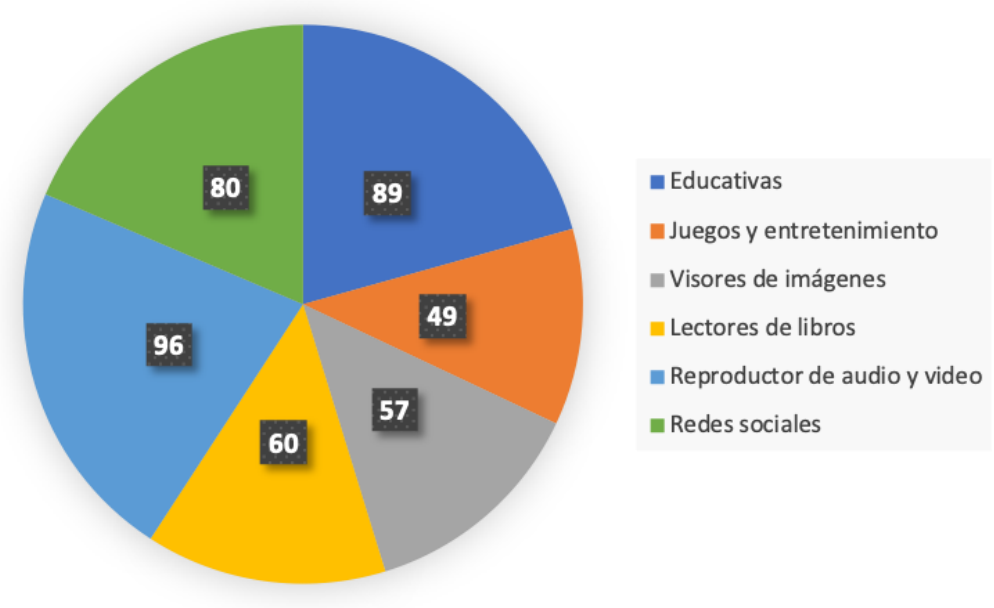

Fuente: Elaboración propia

De igual forma, en el análisis se pudo identificar el porcentaje del tiempo en horas que un profesor de inglés pasa conectado a Internet. El mayor tiempo fue de tres horas al día (22\%) y el menor tiempo fue de una hora (11\%) (ver figura 3 ).

Figura 3. Porcentaje del tiempo en horas que usan el internet los profesores de inglés

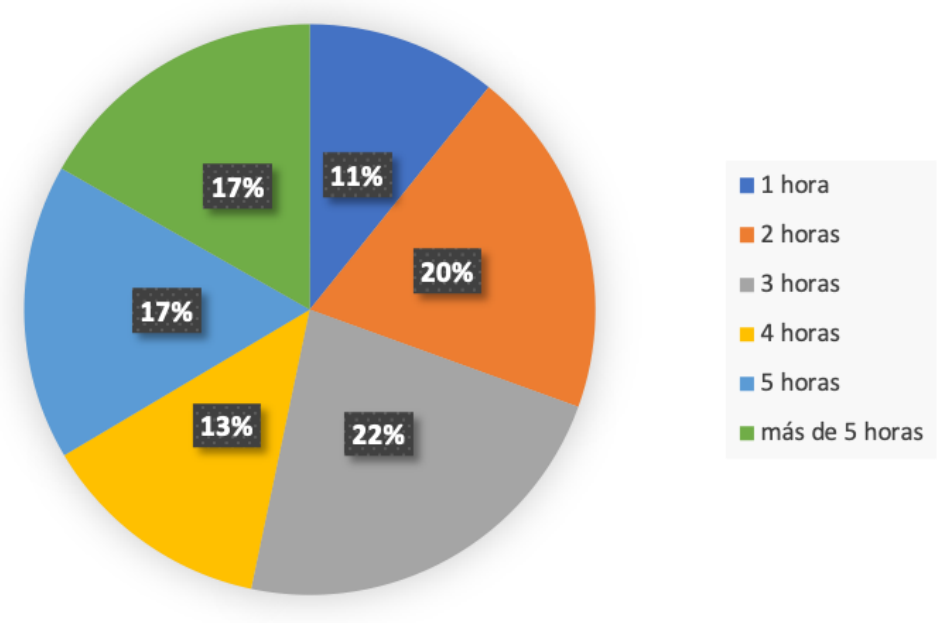

Fuente: Elaboración propia

Ahora bien, teniendo como base las respuestas de los profesores de inglés de enseñanza primaria, se presentan los resultados estadísticos de las dimensiones técnica, pedagógica, comunicación y actitud que integraron el constructo de competencia digital.

En primer lugar, se realizó un análisis de frecuencia y porcentaje por dimensión. El resultado obtenido se analizó teniendo en cuenta el siguiente criterio: si menos de $70 \%$ de las respuestas de los profesores al aspecto valorado se ubicaban en un nivel alto de la escala (competente y muy competente), se recomendaría que el aspecto se trabajara mediante capacitación, ya que se considera como una necesidad para ser atendida. En segundo lugar, y con el fin de consolidar el análisis anterior, se determinó de manera específica el nivel de la 
competencia digital por cada dimensión; para esto fue necesario construir indicadores en escala del 0 al 100. Dichos indicadores se construyeron en función de las puntuaciones mínimas y máximas de cada una de las secciones del cuestionario, y se calcularon a partir de la siguiente fórmula:

$$
\text { Indicador }=\left(\frac{\text { Puntuación de la sección }- \text { Puntuación mínima }}{\text { Rango }}\right) \times 100
$$

Después, se crearon tres niveles para categorizar la competencia digital, a saber: si la puntuación era menor de 60, se consideraría la competencia digital del profesor en un nivel bajo; si la puntuación era entre 60 y 79, la competencia digital se consideraría en un nivel medio, y si la puntuación era mayor de 80 se consideraría en un nivel alto (ver figura 4).

Figura 4. Niveles definidos para los indicadores del 0 al 100

$\left.\left.\sum \begin{array}{c}\text { Nivel bajo } \\ 0-60\end{array} \quad \begin{array}{c}\text { Nivel medio } \\ 61-79\end{array}\right\rangle \begin{array}{c}\text { Nivel alto } \\ 80-100\end{array}\right\rangle$

Fuente: Elaboración propia

A continuación, se presentan los resultados de la dimensión técnica del constructo competencia digital. En la tabla 3 se puede observar que la mayoría de los aspectos está por debajo de $70 \%$, excepto los que se refieren a búsqueda de información en Internet, ofimática y conectividad de equipo periférico. 
Tabla 3. Análisis de frecuencias y porcentajes de la dimensión técnica

$\left.\begin{array}{|l|c|}\hline \text { Aspectos } & \begin{array}{c}\text { Escala alta } \\ \%\end{array} \\ \hline \begin{array}{l}\text { Busca información en Internet con diferentes navegadores, por ejemplo: } \\ \text { Explorer, Chrome, Mozilla, Opera. }\end{array} & 93 \\ \hline \begin{array}{l}\text { Realiza un documento escrito con un procesador de texto, por ejemplo: } \\ \text { Google Docs, Microsoft Word. }\end{array} & 89 \\ \hline \begin{array}{l}\text { Puede conectar equipos de cómputo y audiovisuales (video proyector, } \\ \text { reproductor de películas y audio, bocinas, etcétera). }\end{array} & 84 \\ \hline \begin{array}{l}\text { Crea presentaciones multimedia e interactivas mediante algún programa, por } \\ \text { ejemplo: PowerPoint, Prezi, Emaze, PowToon, etc. }\end{array} & 48 \\ \hline \begin{array}{l}\text { Utiliza las redes sociales como apoyo a sus asignaturas, por ejemplo: } \\ \text { Facebook, Instagram, Pinterest. }\end{array} & 40 \\ \hline \begin{array}{l}\text { Crea videos para sus asignaturas utilizando algún editor de video, por } \\ \text { ejemplo: YouTube Video Editor. }\end{array} & 31 \\ \hline \text { Maneja alguna plataforma, por ejemplo: Moodle, Edmodo, etc. } \\ \hline \text { Crea o edita imágenes mediante algún programa de diseño gráfico, por }\end{array}\right)$

Fuente: Elaboración propia

Por lo evidenciado, se recomienda capacitar a los profesores de inglés en la implementación de las herramientas web 2.0 en sus clases. También, con el fin de que desarrollen su competencia digital, se sugiere motivarlos para que diseñen sus propios recursos didácticos utilizando las TIC. Además, el indicador de valoración en esta dimensión fue de 57 puntos; teniendo en cuenta la categorización del 0 al 100, se trata de un nivel bajo.

En la tabla 4 se presentan los resultados de la dimensión pedagógica. Ahí es posible observar que la mayoría de los aspectos está por debajo de $70 \%$, excepto la que se refiere a fomentar los diferentes estilos de aprendizaje para el idioma inglés (visual, auditivo y kinestésico). 
Tabla 4. Análisis de frecuencias y porcentajes de la dimensión pedagógica

\begin{tabular}{|l|c|}
\hline Aspectos & $\begin{array}{c}\text { Escala alta } \\
\%\end{array}$ \\
\hline $\begin{array}{l}\text { Promueve diferentes estilos de aprendizaje para el idioma inglés utilizando } \\
\text { las TIC, por ejemplo: visual, auditivo y kinestésico. }\end{array}$ & 76 \\
\hline $\begin{array}{l}\text { Implementa diferentes estrategias de enseñanza basadas en TIC que } \\
\text { favorecen el aprendizaje del idioma inglés, por ejemplo: implementación de } \\
\text { juegos interactivos para el aprendizaje de vocabulario en inglés. }\end{array}$ & 64 \\
\hline $\begin{array}{l}\text { Sintetiza la información mediante tablas, gráficos o esquemas para } \\
\text { presentar información a sus estudiantes. }\end{array}$ & 62 \\
\hline $\begin{array}{l}\text { Identifica necesidades en la enseñanza del idioma inglés que pueden ser } \\
\text { abordadas con el uso de las TIC. }\end{array}$ & 53 \\
\hline $\begin{array}{l}\text { Explica las ventajas y limitaciones que presentan las TIC para la enseñanza } \\
\text { del idioma inglés. }\end{array}$ & 50 \\
\hline $\begin{array}{l}\text { Diseña diferentes tipos de evaluación (diagnóstica, formativa y sumativa) } \\
\text { para valorar el aprendizaje en el idioma inglés por medio de test en línea, } \\
\text { por ejemplo: Hot potatoes, ThatQuiz, Google Forms. }\end{array}$ & 22 \\
\hline $\begin{array}{l}\text { Utiliza la videoconferencia como apoyo para el aprendizaje del idioma } \\
\text { inglés, por ejemplo: platicar con estudiantes nativos en el idioma. }\end{array}$ & \\
\hline
\end{tabular}

Fuente: Elaboración propia

A partir de lo comentado líneas arriba, es importante que los profesores utilicen herramientas tecnologías para la elaboración de evaluaciones en línea y gestión de conversaciones virtuales con nativos del idioma inglés. Igualmente, se sugiere que diseñen sus propias estrategias de enseñanza basadas en TIC para que apoyen el desarrollo de las diversas habilidades del inglés (escuchar, hablar, leer y escribir) de una forma dinámica e innovadora.

El indicador de valoración por parte del profesor de inglés en esta dimensión fue de 63 puntos; una vez más, teniendo en cuenta la categorización del 0 al 100, se trata de un nivel bajo.

En la siguiente tabla se presentan los resultados de la dimensión de comunicación. Del análisis de la tabla 5 se puede observar que todos los aspectos están por debajo de $70 \%$. 
Tabla 5. Análisis de frecuencias y porcentajes de la dimensión comunicación

\begin{tabular}{|l|c|}
\hline Aspectos & $\begin{array}{c}\text { Escala alta } \\
\%\end{array}$ \\
\hline $\begin{array}{l}\text { Utiliza programas de la web 2.0 (por ejemplo: Google Drive, YouTube, } \\
\text { wikis, blogs, etc.) para compartir información académica en la red con sus } \\
\text { compañeros profesores y estudiantes. }\end{array}$ & 43 \\
\hline $\begin{array}{l}\text { Crea espacios de práctica del idioma inglés fuera del salón de clase a través } \\
\text { del uso de las TIC. Por ejemplo: actividades o juegos en páginas web } \\
\text { educativas, proponer algún clip de video o película en inglés, diseñar } \\
\text { actividades online, etc. }\end{array}$ & 40 \\
\hline $\begin{array}{l}\text { Utiliza el correo electrónico para propiciar la práctica del idioma inglés (por } \\
\text { ejemplo: pen pals, escribir textos para practicar lectura y escritura) o para } \\
\text { enviar tareas. }\end{array}$ & 33 \\
\hline $\begin{array}{l}\text { Se comunica con estudiantes por medio de alguna plataforma educativa fuera } \\
\text { del horario escolar, por ejemplo: foro, chat, wiki, etc. }\end{array}$ & 21 \\
\hline
\end{tabular}

Fuente: Elaboración propia

Como se puede percibir en la tabla 5, es recomendable capacitar a los profesores de inglés para el trabajo con tecnología que permita la interacción y gestión de los procesos de enseñanza y aprendizaje en modalidades semipresenciales y a distancia. Al igual que en los casos anteriores, el indicador de valoración en esta dimensión fue bajo, ya que registró 42 puntos.

En la siguiente tabla se presentan los resultados de la dimensión actitud. Del análisis de la tabla 6 se puede observar que la mayoría de los aspectos están por encima de $70 \%$, excepto las que tienen que ver con fomentar en los estudiantes el uso de las TIC y el aspecto ético al momento de utilizar información obtenida de internet. 
Tabla 6. Análisis de frecuencias y porcentajes de la dimensión actitud

\begin{tabular}{|l|c|}
\hline Aspectos & $\begin{array}{l}\text { Escala alta } \\
\%\end{array}$ \\
\hline Disposición al aprendizaje continuo y a la actualización permanente. & 85 \\
\hline $\begin{array}{l}\text { Evita el acceso a la información conflictiva o ilegal que pueda generar } \\
\text { problemas. }\end{array}$ & 80 \\
\hline $\begin{array}{l}\text { Desarrolla una actitud abierta y crítica ante las nuevas tecnologías } \\
\text { (contenidos, entretenimiento, etc.). }\end{array}$ & 68 \\
\hline $\begin{array}{l}\text { Demuestra aspectos éticos y legales al momento de utilizar la información } \\
\text { obtenida de Internet, por ejemplo: propiedad intelectual, licencias de } \\
\text { distribución. }\end{array}$ & 47 \\
\hline $\begin{array}{l}\text { Fomenta en sus estudiantes el uso de las TIC al pedirles que realicen tareas } \\
\text { que suponga usarlas (un video, una grabación, un blog, etc.). }\end{array}$ & \\
\hline
\end{tabular}

\section{Fuente: Elaboración propia}

Es necesario que los profesores de inglés diseñen y desarrollen estrategias de enseñanza que fomenten en sus estudiantes el uso de las TIC; también es vital que conozcan e implementen el código de ética al momento de utilizar información que se obtiene de internet. Se debe agregar a lo comentado que el indicador de valoración por parte del profesor de inglés en esta dimensión fue de 74 puntos. A diferencia del resto, este fue considerado en un nivel medio.

En una tercera fase de los análisis estadísticos y con la idea de contestar el segundo objetivo del estudio, se identificaron las variables que influyen en la competencia digital de los profesores del idioma inglés. En primer lugar, se determinó si existía diferencia significativa entre la variable sexo y la competencia digital de los profesores de inglés de enseñanza primaria. Para ello se realizó una prueba $t$ para muestras independientes. En la tabla 7 se observa que no existe diferencia entre la competencia digital de los profesores de inglés con la variable sexo ( $p$ $>0.05$ ).

Tabla 7. Comparación de la competencia digital con la variable sexo

\begin{tabular}{|c|c|c|c|c|}
\hline \multicolumn{5}{|c|}{ Variable sexo } \\
\cline { 1 - 3 } Sexo & $\bar{x}$ & D.S. & $t$ & $p$ \\
\hline Hombre & 59.1 & 16.7 & .561 & .577 \\
\cline { 1 - 3 } Mujer & 61.3 & 19.3 & & \\
\hline
\end{tabular}

Fuente: Elaboración propia 


\section{Referencias}

Arnau, J. (1995). Metodologías cuantitativas en la investigación psicológica. Barcelona, España: Experimental.

Arteaga, C. (2011). Uso de las TIC para el aprendizaje del inglés en la Universidad Autónoma de Aguascalientes. Apertura, 3(2). Recuperado de http://www.udgvirtual.udg.mx/apertura/index.php/apertura/article/view/206/221.

Bauman, Z. (2004). Modernidad líquida. México: Fondo de Cultura Económica.

Butler, M. (2011). The history of CALL: The intertwining paths of technology and second/foreign language teaching. International Journal of Computer-Assisted Language Learning and Teaching, 1(1). Retrieved from https://www.igiglobal.com/journal/international-journal-computer-assisted-language/41023.

Buzzetto, N. (2012). Social Networking in Undergraduate Education. Interdisciplinary Journal of Information, Knowledge, and Management, 7(1), 63-90. Retrieved from http://www.ijikm.org/Volume7/IJIKMv7p063-090Buzzetto611.pdf.

Cabero, J., Llorente, M. del C. y Marín, V. (2010). Hacia el diseño de un instrumento de diagnóstico "competencias tecnológicas del profesorado universitario". Revista Iberoamericana de Educación, 7(52). Recuperado de https://core.ac.uk/download/pdf/51388280.pdf.

Cabrera, J., Sánchez, I. y Rojas, F. (2016). Uso de objetos virtuales de aprendizaje OVAS como estrategia de enseñanza-aprendizaje inclusivo y complementario a los cursos teóricosprácticos. Educación en Ingeniería, 11(22). Recuperado de https://www.educacioneningenieria.org/index.php/edi/article/view/602.

Cajar, M. y Rojas, B. (2015). Influencia de las TIC en el desarrollo de competencias comunicativas del idioma inglés en los estudiantes del grado quinto de la institución educativa Montessori sede primaria de Pitalito - Huila 2014. (tesis maestría). Universidad Norbert Wiener, Pitalito (Huila).

Casal, J. y Mateu, E. (2003). Tipos de muestreo. Revista de Epidemiología y Medicina Preventiva, 1(1), 3-7.

Cea D'Ancona, M. (2001). Metodología cuantitativa. Estrategias y técnicas de investigación social. Madrid, España: Síntesis.

Cozby, P. and Bates, S. (2015). Methods in Behavioral Research (12 ${ }^{\text {th }}$ ed.). New York, United States: McGraw-Hill Education.

Chacón, C. y Pérez, C. (2011). El podcast como innovación en la enseñanza del idioma inglés como lengua extranjera. Pixel-Bit. Revista de Medios y Educación, (39), 41-54. Recuperado de https://recyt.fecyt.es/index.php/pixel/article/viewFile/61449/37462. 


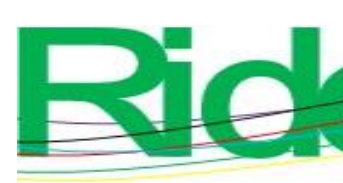

Revista Iberoamericana para la

Investigación y el Desarrollo Educativo

ISSN $2007-7467$

Ibrahim, A. (2010). Information \& Communication Technologies in ELT. Journal of Language Teaching and Research, 1(3), 211-214. Retrieved from http://dx.doi.org/10.4304/jltr.1.3.211-214.

Isaac, S. and Michael, W. (1995). Handbook in Research and Evaluation. San Diego, United States: EDITS Publishers.

International Society for Technology in Education [ISTE]. (2008). ISTE Standards for Educators. Retrieved from http://www.iste.org/standards/iste-standards/standards-forteachers.

Lorber, M. (1977). Objectives, methods and evaluation for secondary teaching. New Jersey, Pearson.

Morchio, M. (2014). El rol de las TIC en la clase de inglés. Ponencia presentada en el Congreso Iberoamericano de Ciencia, Tecnología, Innovación y Educación. Buenos Aires, del 12 al 14 de noviembre de 2014. Recuperado de www.oei.es/historico/congreso2014/memoriactei/753.pdf.

Moreno, T. (2011). Didáctica de la Educación Superior: nuevos desafíos en el siglo XXI. Perspectiva Educacional, 50(2). Recuperado de https://www.redalyc.org/articulo.oa?id=333327290003.

Mortis, S., Cuervo, A., Armenta, J., López, R. y Salazar, O. (2013). Competencias digitales en docentes de educación secundaria. Municipio de un Estado del Noroeste de México. Perspectiva Educacional, Formación de Profesores, 52(2), 135-153. Recuperado de https://www.redalyc.org/articulo.oa?id=333328170007.

Páez, V. (2001). El profesor de idiomas: sus cualidades y competencias. Revista Comunicación, 11(3). Recuperado de https://www.redalyc.org/pdf/166/16611306.pdf.

Pérez, C. y Monteza, C. (2013). Nuevos problemas del aprendizaje en la era digital. Competencias digitales y nuevas formas de aprender. Actualidades pedagógicas, (61). Recuperado de: https://ciencia.lasalle.edu.co/cgi/viewcontent.cgi?article=1208\&context=ap

Perrenoud, P. (2004). Diez nuevas competencias para enseñar. Querétaro, México. Recuperado de https://www.uv.mx/dgdaie/files/2013/09/Philippe-Perrenoud-Diez-nuevascompetencias-para-ensenar.pdf.

Prensky, M. (2010) Nativos e inmigrantes digitales. Institución Educativa SEK. Recuperado de https://www.marcprensky.com/writing/Prensky-

NATIVOS\%20E\%20INMIGRANTES\%20DIGITALES\%20(SEK).pdf. 
